\title{
Fuzzy Logic Deadzone Compensation with Feedback Linearization of Nonlinear Systems
}

\author{
Jun Oh Jang \\ Department of Software Engineering, Uiduk University, Kyongju, South Korea \\ Email: jojang@uu.ac.kr
}

How to cite this paper: Jang, J.O. (2019) Fuzzy Logic Deadzone Compensation with Feedback Linearization of Nonlinear Systems. Applied Mathematics, 10, 87-99. https://doi.org/10.4236/am.2019.103008

Received: February 23, 2019

Accepted: March 18, 2019

Published: March 21, 2019

Copyright $\odot 2019$ by author(s) and Scientific Research Publishing Inc. This work is licensed under the Creative Commons Attribution International License (CC BY 4.0).

http://creativecommons.org/licenses/by/4.0/

\begin{abstract}
A fuzzy logic compensator is designed for feedback linearizable nonlinear systems with deadzone nonlinearity. The classification property of fuzzy logic systems makes them a natural candidate for the rejection of errors induced by the deadzone, which has regions in which it behaves differently. A tuning algorithm is given for the fuzzy logic parameters, so that the deadzone compensation scheme becomes adaptive, guaranteeing small tracking errors and bounded parameter estimates. Formal nonlinear stability proofs are given to show that the tracking error is small. The fuzzy logic deadzone compensator is simulated on a one-link robot system to show its efficacy.
\end{abstract}

\section{Keywords}

Deadzone Compensation, Feedback Linearization, Fuzzy Logic, Stability

\section{Introduction}

Very accurate control is required in mechanical devices such as $x y$ positioning tables [1], overhead crane mechanisms [2], robot manipulators [3], etc. For many of these devices, the performance is limited by deadzone, friction, and backlash. Precise positioning, in particular, control of very small displacement is an especially difficult problem for micro positioning devices. Due to the nonanalytic nature of the actuator nonlinearities and the fact that their exact parameters (e.q. width of deadzone) are unknown, such systems present a challenge for the control design engineer. A number of control strategies have been developed to overcome the problems caused by the deadzone effects. The most relevant published strategies are: deadzone compensation using neural networks [4] and variable structure control [5]. Control of gear transmission servo system with asymmetric deadzone nonlinearity is proposed in [6] and [7]. Compensa- 
tion for nonsymmetrical deadzones is considered in [8] for nonlinear systems in Brunosky form with known nonlinear functions, and for unknown nonlinear canonical form systems in [9] where a backstepping approach is used. The use of fuzzy logic systems has accelerated in recent years in many areas, including feedback control [10]. Fuzzy logic deadzone compensation schemes are provided in [11] [12] [13]. Particularly important in fuzzy logic control are the universal function approximation capabilities of fuzzy logic systems [14] [15]. The fuzzy logic systems offer significant advantages over adaptive control, including no requirement for linearity in the parameters assumptions and no need to compute a regression matrix for each specific system. Actuator nonlinearities are typically defined in terms of piecewise linear functions according to the region to which the argument belongs. The fuzzy logic function approximation properties and ability of fuzzy logic systems to discriminate information based on regions of the input variables, make them an ideal candidate for compensation of non-analytic actuator nonlinearities.

In this paper, we present the deadzone compensation method for feedback linearizable nonlinear systems. In Section 2, a brief overview on the feedback linearization theory is given [16] [17]. The fuzzy deadzone compensation technique is described in Section 3. An adaptive fuzzy deadzone compensation scheme combined with a feedback linearizing control law is developed and analyzed in Section 4. Also, a practical bound on the tracking error from the tracking error dynamics is derived. The zero dynamics systems caused by joint damping are analyzed and shown to be bounded-input, bounded-state stable. Section 5, the fuzzy deadzone compensation is applied to control a single link manipulator with deadzone nonlinearity as well as joint flexibility and damping. In addition, we investigate the performance of the fuzzy logic deadzone compensation through the computer simulations.

\section{Feedback Linearization}

Feedback linearization has been proved to be a powerful tool in control of nonlinear systems. The Lie derivative [18] of two functions $h(x) \in R$ and

$$
\begin{gathered}
f(x)=\left[f_{1}(x), f_{2}(x), \cdots, f_{n}(x)\right]^{\mathrm{T}} \in R^{n}, \text { for } x \in R^{n} \text {, is defined as } \\
L_{f} h=\frac{\partial h}{\partial x_{1}} f_{1}+\cdots+\frac{\partial h}{\partial x_{n}} f_{n} .
\end{gathered}
$$

For $g(x) \in R^{n}$, and recursively with $L_{f}^{0} h=h$, it follows that

$$
\begin{gathered}
L_{g} L_{f} h(x)=\frac{\partial L_{f} h}{\partial x} g \\
L_{f}^{i+1} h=L_{f}\left(L_{f}^{i} h\right)=\frac{\partial L_{f}^{i} h}{\partial x} f, \quad i=1,2, \cdots
\end{gathered}
$$

A single input single output nonlinear system

$$
\begin{aligned}
& \dot{x}=f(x)+g(x) u, x \in R^{n}, u \in R \\
& y=h(x), y \in R
\end{aligned}
$$


has relative degree $\rho$ at a point $x_{0}$ if 1) $L_{g} L_{f}^{k} h(x)=0$ for $\forall x \in N\left(x_{0}\right)$ (a neighborhood of $\left.x_{0}\right), \forall k=0,1,2, \cdots, \rho-2$ and 2) $L_{g} L_{f}^{\rho-1} h\left(x_{0}\right) \neq 0$.

Assume that the system (4) has relative degree $n$ at $x$ and define

$$
z=T(x)=\left[\begin{array}{c}
T_{1}(x) \\
T_{2}(x) \\
T_{3}(x) \\
\vdots \\
T_{n}(x)
\end{array}\right]=\left[\begin{array}{c}
h(x) \\
L_{f} h(x) \\
L_{f}^{2} h(x) \\
\vdots \\
L_{f}^{n-1} h(x)
\end{array}\right]
$$

which is a diffeomorphism. It follows that

$$
\begin{aligned}
& \dot{T}_{k-1}=T_{k}, k=2,3, \cdots, n \\
& \dot{T}_{n}=L_{f}^{n} h(x)+L_{g} L_{f}^{n-1} h(x) u
\end{aligned}
$$

With the new state $z=T(x)$, the feedback control law

$$
u=\frac{1}{L_{g} L_{f}^{n-1} h(x)}\left(r-L_{f}^{n} h(x)\right)
$$

linearizes the system (4), i.e., which leads to the system

$$
\begin{aligned}
& \dot{z}_{i}=z_{i+1}, i=1,2, \cdots, n-1 \\
& \dot{z}_{n}=r
\end{aligned}
$$

which can be put in a matrix form $\dot{z}=A z+b r$, where $r \in R$ is a new input. Here we note that $T(x)$ should be a diffeomorphism in the region, which is reachable by $x(t)$ driven by $r(t)$.

If the system (4) has relative degree $\rho<n$ at $x$, the transformed system becomes

$$
\begin{aligned}
& \dot{z}_{1}=z_{2}, \dot{z}_{2}=z_{3}, \cdots, \dot{z}_{\rho-1}=z_{\rho} \\
& \dot{z}_{\rho}=b\left(\xi, \xi_{0}\right)+a\left(\xi, \xi_{0}\right) u, \dot{\xi}_{0}=q\left(\xi, \xi_{0}\right)
\end{aligned}
$$

where $\xi=\left[z_{1}, z_{2}, \cdots, z_{\rho}\right]^{\mathrm{T}}$ and $\xi_{0}=\left[z_{\rho+1}, \cdots, z_{n}\right]^{\mathrm{T}}$. The zero dynamics system is $\dot{\xi}_{0}=q\left(\xi, \xi_{0}\right)$ [18] [19]. A feedback linearizing controller $u=\left(1 / a\left(\xi, \xi_{0}\right)\right)\left(r-b\left(\xi, \xi_{0}\right)\right)$ may ensure that $\xi$ is bounded. However, even if the system zero dynamics $\dot{\xi}_{0}=q\left(0, \xi_{0}\right)$ are stable, a bounded $\xi$ may not ensure that $\xi_{0}$ is also bounded. For a tracking problem $z_{1}, z_{2}, \cdots, z_{\rho}$ do not converge to zero. The zero dynamics subsystem is driven by the "inputs" $z_{i}$, $i=1,2, \cdots, \rho$, with its own states as $z_{j}, j=\rho+1, \rho+2, \cdots, n$. For bounded inputs $z_{i}, i=1,2, \cdots, \rho$, and with arbitrary initial conditions $z_{j}(0)$, for $z_{j}(t)$ to be bounded, the condition is that the zero dynamics subsystem $\dot{z}_{j}(t)=q_{j}(z(t))$, $j=\rho+1, \rho+2, \cdots, n$, is bounded input, bound-state stable [19].

\section{Compensation of Deadzone Nonlinearity}

In this section a fuzzy logic precompensator is designed for the non-symmetric deadzone nonlinearity. It is shown that the fuzzy logic approach includes and subsumes approaches based on switching logic and indicator functions [7] [8]. 
This brings these references very close to fuzzy logic work in [11], and potentially allows for more exotic compensation schemes for actuator nonlinearities using more complex decision (e.g. membership) functions. This section provides a rigorous framework for fuzzy logic applications in deadzone compensation for feedback linearization systems.

If $u, v$ are scalars, the nonsymmetric deadzone nonlinearity, shown in Figure 1 , is given by

$$
u=N(v)=\left\{\begin{array}{lc}
v+d_{-}, & v<-d_{-} \\
0, & -d_{-} \leq v<d_{+} \\
v-d_{+}, & d_{+} \leq v
\end{array}\right.
$$

The parameter vector $d=\left[d_{+} d_{-}\right]^{\mathrm{T}}$ characterizes the width of the system deadband. In practical control systems the width of the deadzone is unknown, so that compensation is difficult. Most compensation schemes cover only the case of symmetric deadzones where $d_{-}=d_{+}$.

The nonsymmetric deadzone may be written as

$$
u=N(v)=v-\operatorname{sat}_{d}(v)
$$

where the nonsymmetric saturation function is defined as

$$
\operatorname{sat}_{d}(v)=\left\{\begin{array}{lr}
-d_{-}, & v<-d_{-} \\
u, & -d_{-} \leq v<d_{+} \\
d_{+}, & d_{+} \leq v
\end{array}\right.
$$

To offset the deleterious effects of deadzone, one may place a precompensator as illustrated in Figure 2. There, the desired function of the precompensator is

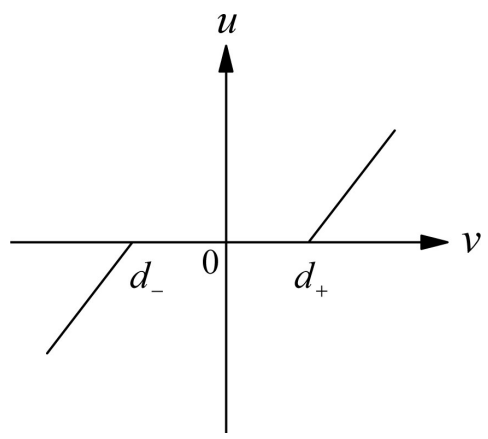

Figure 1. Deadzone nonlinearity.

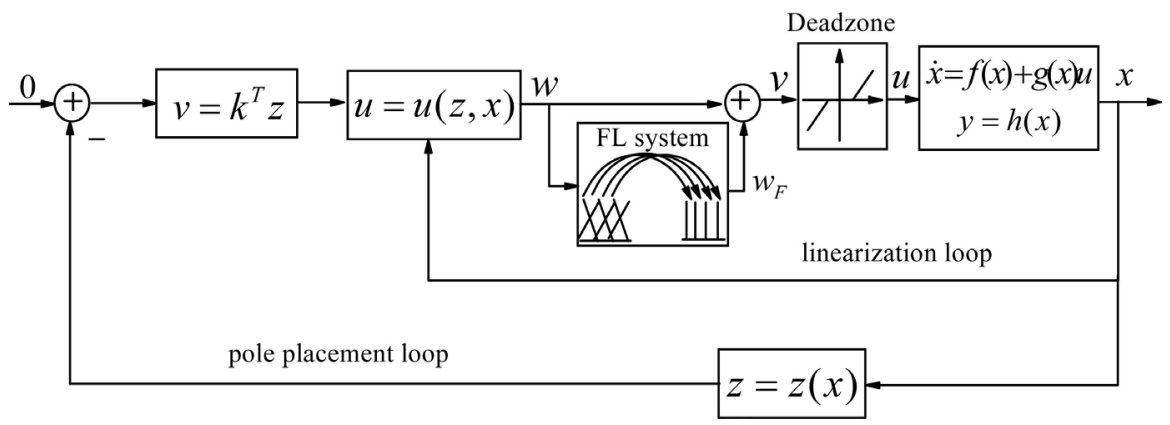

Figure 2. Fuzzy deadzone compensation with feedback linearization. 
to cause the composite throughput from $w$ to $u$ to be unity. The power of fuzzy logic systems is to that they allow one to use intuition based on experience to design control systems, then provide the mathematical machinery for rigorous analysis and modification of the intuitive knowledge, for example, through learning or adaptation, to give guaranteed performance, as will be shown in Section 4. Due to the fuzzy logic classification property, they are particularly powerful when the nonlinearity depends on the region in which the argument $v$ of the nonlinearity is located, as in the non-symmetric deadzone.

A deadzone precompensator using engineering experience would be discontinuous and depend on the region within which $w$ occurs. It would be naturally described using the rules

$$
\begin{aligned}
& \text { If }(w \text { is positive }) \text { then }\left(v=w+\hat{d}_{+}\right) \\
& \text {If ( } w \text { is negative) then }\left(v=w-\hat{d}_{-}\right)
\end{aligned}
$$

where $\hat{d}=\left[\begin{array}{ll}\hat{d}_{+} & \hat{d}_{-}\end{array}\right]^{\mathrm{T}}$ is an estimate of the deadzone width parameter vector $d$.

To make this intutive notion mathematically precise for analysis define the membership function's

$$
\begin{aligned}
& X_{+}(w)= \begin{cases}0, & w<0 \\
1, & 0 \leq w\end{cases} \\
& X_{-}(w)= \begin{cases}1, & w<0 \\
0, & 0 \leq w\end{cases}
\end{aligned}
$$

One may write the precompensator as

$$
v=w+w_{F}
$$

where $w_{F}$ is given by the rule base.

$$
\begin{aligned}
& \text { If }\left(w \in X_{+}(w)\right) \text { then }\left(w_{F}=\hat{d}_{+}\right) . \\
& \qquad \text { If }\left(w \in X_{-}(w)\right) \text { then }\left(w_{F}=-\hat{d}_{-}\right) .
\end{aligned}
$$

The output of the fuzzy logic system with this rule base is given by

$$
w_{F}=\frac{\hat{d}_{+} X_{+}(w)-\hat{d}_{-} X_{-}(w)}{X_{+}(w)+X_{-}(w)} .
$$

The estimates $\hat{d}_{+},-\hat{d}_{-}$are, respectively, the control representive value of $X_{+}(w)$ and $X_{-}(w)$. This may be written (note $X_{+}(w)+X_{-}(w)=1$ ) as

$$
w_{F}=\hat{d}^{\mathrm{T}} X(w)
$$

where the fuzzy logic basis function vector given by

$$
X(w)=\left[\begin{array}{c}
X_{+}(w) \\
-X_{-}(w)
\end{array}\right]
$$

is easily computed given any value of $w$.

It should be noted that the membership functions (14) are the indicator functions and $X(w)$ is similar to the regressor [6] [7] [8] [9]. The composite through from $w$ to $u$ of the fuzzy logic compensator plus the deadzone is 


$$
u=N(v)=N\left(w+w_{F}\right)=w+\left[w_{F}-s a t_{d}\left(w+w_{F}\right)\right] .
$$

The fuzzy logic compensator may be expressed as follows

$$
v=w+w_{F}=w+\hat{d}^{\mathrm{T}} X(w)
$$

where $\hat{d}$ is estimated deadzone widths.

Given the fuzzy logic compensator with rulebase (16), the throughput of the compensator plus deadzone is given by

$$
u=w+\tilde{d}^{\mathrm{T}} X(w)-\tilde{d}^{\mathrm{T}} \delta
$$

where the deadzone width estimation error is given by

$$
\tilde{d}=d-\hat{d}
$$

and the modeling mismatch term $\delta$ is bounded so that $|\delta|<\delta_{M}$ for some scalar $\delta_{M}$.

\section{Adaptive Fuzzy Logic Deadzone Compensation with Linearization}

In this section we show how to provide fuzzy logic deadzone compensation for deadzone in feedback linearizable nonlinear system. The proposed control structure is shown in Figure 2. The fuzzy logic deadzone compensator is given by (21). We show to tune or learn the deadzone width estimates $\hat{d}$ on-line so that the tracking error is guaranteed small and all internal states are bounded. This turns the deadzone compensator into an adaptive fuzzy logic deadzone compensator.

The system has a deadzone nonlinearity $N(\cdot)$ with control input $v$, that is

$$
\begin{aligned}
& \dot{x}=f(x)+g(x) u, u=N(v) \\
& y=h(x)
\end{aligned}
$$

From the (24) and (22)

$$
\dot{x}=f(x)+g(x)\left(w+\tilde{d} X(w)-\tilde{d}^{\mathrm{T}} \delta\right) .
$$

The control task now is to design a feedback row of $w$ and a fuzzy logic logic tuning law for $\tilde{d}$ to ensure desired closed loop system properties.

1) Design with exact feedback linearization [20]: In this case, the assumption is that the system (25) has relative degree $n$. Introduce the $z=T(x) \in R^{n}$, where $T(x)$ is a diffeomorphism defined in (5). Then it follows that

$$
\dot{z}=\frac{\partial T}{\partial x}\left(f(x)+g(x) w+g(x)\left(\tilde{d}^{\mathrm{T}} X(w)-\tilde{d}^{\mathrm{T}} \delta\right)\right) .
$$

Choose the feedback linearizing control input $w$ as

$$
w=\frac{1}{L_{g} T_{n}}\left(r-L_{f} T_{n}\right)=\alpha^{-1}(x)(r-\beta(x))
$$

where $T_{n}(x)$ is the last row of $T(x), \alpha(x)=L_{g} T_{n}$, and $\beta(x)=L_{f} T_{n}$. This control law has the properties 


$$
\begin{gathered}
\frac{\partial T}{\partial x}\left(f(x)-g(x) \alpha^{-1}(x) \beta(x)\right)=A z \\
\frac{\partial T}{\partial x} g(x) \alpha^{-1}(x)=b
\end{gathered}
$$

where $A$ and $b$ are from (8).

With the controller (27), we can rewrite (26) as

$$
\dot{z}=A z+b r+\frac{\partial T}{\partial x} g(x)\left(\tilde{d}^{\mathrm{T}} X(w)-\tilde{d}^{\mathrm{T}} \delta\right) .
$$

Then we choose the linear feedback control law

$$
r=-F z+r_{d}
$$

where $F \in R^{1 \times n}$ is such that $A-b F$ has desired stable eigenvalues and $r_{d}$ is a reference input, and introduce the reference system as

$$
\dot{z}_{m}=(A-b F) z_{m}+b r_{d} .
$$

For the tracking error $\tilde{z}=z-z_{m}$, it follows from (30)-(32) that

$$
\dot{\tilde{z}}=\dot{z}-\dot{z}_{m}=(A-b F) \tilde{z}+\frac{\partial T}{\partial x} g(x)\left(\tilde{d}^{\mathrm{T}} X(w)-\tilde{d}^{\mathrm{T}} \delta\right)
$$

Theorem 1: Given the system (24), select the tracking control (31) and deadzone compensator (21), where $X(w)$ is given by (19). The estimated deadzone widths be provided by the fuzzy logic system tuning algorithm

$$
\dot{\hat{d}}(t)= \begin{cases}\alpha(x) \Gamma X(w) \tilde{z}^{\mathrm{T}} P b & \text { if } \delta=0 \\ \alpha(x) \Gamma\left(X(w) \tilde{z}^{\mathrm{T}}-k \hat{d}\|\tilde{z}\|\right) P b & \text { if } \delta \neq 0\end{cases}
$$

where the scalar $k>0$ and $\Gamma$ is chosen dialgonal with positive diagonal elements. If $\delta=0$, then all closed loop signals are bounded. If $\delta \neq 0$, The tracking error evolves with a practical bound

$$
\|\tilde{z}\| \leq \frac{\alpha(x) P b c_{0}^{2}}{4 Q_{\min } k} .
$$

where $Q_{\min }$, minimum singular value of $Q$.

Proof: Define a Lyapunov function candidate for error dynamics (33) as

$$
V(\tilde{z}, \tilde{d})=\frac{1}{2}\left(\tilde{z}^{\mathrm{T}} P \tilde{z}+\tilde{d}^{\mathrm{T}} \Gamma^{-1} \tilde{d}\right)
$$

where $P \in R^{n \times n}$ with $P=P^{\mathrm{T}}>0$ satisfies the Lyapunov equation $P(A-b F)+(A-b F)^{\mathrm{T}} P=-2 Q$ for a chosen $n \times n$ matrix $Q=Q^{\mathrm{T}}>0$.

Differentiating (36) and using (29) and (33) yields

$$
\dot{V}(t)=-\tilde{z}^{\mathrm{T}}(t) Q \tilde{z}(t)+\alpha(x) \tilde{z}^{\mathrm{T}}(t) P b\left(\tilde{d}^{\mathrm{T}} X(w)-\tilde{d}^{\mathrm{T}} \delta\right)+\tilde{d}^{\mathrm{T}} \Gamma^{-1} \dot{\tilde{d}} .
$$

If $\delta=0$, the tuning algorithm (34) results in $\dot{V}=-\tilde{z}^{\mathrm{T}}(t) Q \tilde{z}(t) \leq 0$. This means that $V(t)$ is bounded and $\tilde{z}=z-z_{m} \in L^{2}$, i.e., $\tilde{z}$ and $\tilde{d}$ are bounded, and so $z$ and $d$. Since $z=T(x)$ is a diffeomorphism. $x(t)$ is also bounded, and are $w$ and $\dot{\tilde{z}}$, which implies that $\lim _{t \rightarrow \infty}\left(z(t)-z_{m}(t)\right)=0$. 
If $\delta \neq 0$, substitution the tuning algorithm (34) gives

$$
\begin{aligned}
\dot{V}(t)= & -\tilde{z}^{\mathrm{T}}(t) Q \tilde{z}(t)+\alpha(x) \tilde{z}^{\mathrm{T}}(t) P b\left(\tilde{d}^{\mathrm{T}} X(w)-\tilde{d}^{\mathrm{T}} \delta\right) \\
& +\tilde{d}^{\mathrm{T}} \Gamma^{-1}\left\{-\alpha(x) \Gamma\left(X(w) \tilde{z}^{\mathrm{T}}-k \hat{d}\|\tilde{z}\|\right) P b\right\}
\end{aligned}
$$

where $\tilde{d}=d-\hat{d}$ and $\dot{d}=0$ by deadzone widths is constant, $\dot{\tilde{d}}=-\dot{\hat{d}}$. Therefore,

$$
\begin{aligned}
\dot{V}(t) & =-\tilde{z}^{\mathrm{T}}(t) Q \tilde{z}(t)-\alpha(x) \tilde{z} P b \tilde{d}^{\mathrm{T}} \delta+\tilde{d}^{\mathrm{T}} \alpha(x) k(d-\tilde{d})\|\tilde{z}\| P b \\
& \leq-Q|\tilde{z}|^{2}-\alpha(x)\|\tilde{z}\| P b\|\tilde{d}\| \delta_{M}+\|\tilde{d}\| \alpha(x) k d_{M}\|\tilde{z}\| P b-\alpha(x) k\|\tilde{d}\|^{2}\|\tilde{z}\| P b
\end{aligned}
$$

where $\delta<\delta_{M}, d<d_{M}$ for some scalar $d_{M}, \delta_{M}$ respectively, and bounding properties were used. Therefore

$$
\dot{V}(t) \leq-\|\tilde{z}\|\left[Q_{\min }\|\tilde{z}\|-\alpha(x) P b c_{0}\|\tilde{d}\|+\alpha(x) k\|\tilde{d}\|^{2} P b\right]
$$

where $c_{o}=k d_{M}-\delta_{M}$.

This is negative as long as the quantity in the brace is positive. To determine conditions for this, complete the square to see that $\dot{V}$ is negative as long as either

$$
\|\tilde{z}\|>\frac{\alpha(x) P b c_{0}^{2}}{4 Q_{\min } k}
$$

or

$$
\|\tilde{d}\|>\frac{c_{0}}{k}
$$

According to the standard Lyapunov theorem, the tracking error decreases as long as the error is bigger than the right-hand side of Equation (41). This implies Equation (43) gives a practical bound on the tracking error

$$
\|\tilde{z}\| \leq \frac{\alpha(x) P b c_{0}^{2}}{4 Q_{\min } k} .
$$

Also, Lyapunov extension shows that the deadzone width bound, $\|\tilde{d}\|$, is bounded to a neighborhood of the right hand side of Equation (42).

2) Design for systems with zero dynamics: When the system relative degree is $\rho<n$, the system can only be partially feedback linearized. Let $z=T(x) \in R^{n}$ be a diffeomorphism. In this case, there are two parts of $z=T(x): \xi=T_{c}(x)=\left[z_{1}, \cdots, z_{\rho}\right]^{\mathrm{T}}$, which is the feedback linearization part, and $\xi_{0}=T_{z}(x)=\left[z_{\rho+1}, \cdots, z_{n}\right]^{\mathrm{T}}$, which is the zero dynamics. Similar to (26), we can obtain

$$
\begin{aligned}
\dot{\xi} & =\frac{\partial T_{f}}{\partial x}\left(f(x)+g(x) w+g(x)\left(\tilde{d}^{\mathrm{T}} X(w)-\tilde{d}^{\mathrm{T}} \delta\right)\right) \\
& =A_{\rho} \xi+b_{\rho} r+\frac{\partial T_{c}}{\partial x} g(x)\left(\tilde{d}^{\mathrm{T}} X(w)-\tilde{d}^{\mathrm{T}} \delta\right)
\end{aligned}
$$

by choosing a control law $w=\alpha^{-1}(x)(r-\beta(x))$, which has the properties 


$$
\begin{gathered}
\frac{\partial T_{f}}{\partial x}\left(f(x)-g(x) \alpha^{-1}(x) \beta(x)\right)=A_{\rho} \xi \\
\frac{\partial T_{f}}{\partial x} g(x) \alpha^{-1}(x)=b_{\rho}
\end{gathered}
$$

The zero dynamics system then is $\dot{\xi}_{0}=q\left(\xi, \xi_{0}\right)$. The reference model is similar to that in (32) but $z_{m} \in R^{\rho}$. Defining the new tracking error $\tilde{z}=\xi-z_{m}$; the fuzzy logic tuning algorithm is of the same form as that in (34) which ensures that $\xi$ and $\hat{d}$ are bounded. If the zero dynamics system is bounded input, bounded-state stable, the system state $z$ is bounded, and so is $X$.

\section{Simulation Results}

In this section, we illustrate the effectiveness of fuzzy logic deadzone compensation with feedback linearization by computer simulation. Consider the control of a single link manipulator with joint flexibility and damping as well as with deadzone nonlinearity in Figure 3.

$$
\begin{aligned}
& I \ddot{q}_{1}+M g L \sin q_{1}+k_{e}\left(q_{1}-q_{2}\right)+b\left(\dot{q}_{1}-\dot{q}_{2}\right)=0 \\
& J \ddot{q}_{2}-k_{e}\left(q_{1}-q_{2}\right)-b\left(\dot{q}_{1}-\dot{q}_{2}\right)=u, u=N(v)
\end{aligned}
$$

where $q_{1}, q_{2}$ are the angular positions of the link and motor, $I$ and $J$ are the inertia, $k_{e}$ is the elasticity constant of the joint spring, $M$ and $L$ represent the mass and the position of the center of gravity of the link, and $u$ is the torque applied at the motor. With the state variables $x_{1}=q_{1}, x_{2}=\dot{q}_{1}, x_{3}=q_{2}$, $x_{4}=\dot{q}_{2}$, this system can be expressed as that in (24).

For the system (46) with flexibility only, i.e., $k_{e}>0, b=0$, as in [21], a diffeomorphism $T(x)$ is

$$
\begin{aligned}
& z_{1}=T_{1}(x)=x_{1} \\
& z_{2}=T_{2}(x)=x_{2} \\
& z_{3}=T_{3}(x)=-\frac{M g l}{I} \sin x_{1}-\frac{k_{e}}{I}\left(x_{1}-x_{3}\right) \\
& z_{4}=T_{4}(x)=-\frac{M g l}{I} x_{2} \cos x_{1}-\frac{k_{e}}{I}\left(x_{2}-x_{4}\right)
\end{aligned}
$$

and the feedback linearizing control is

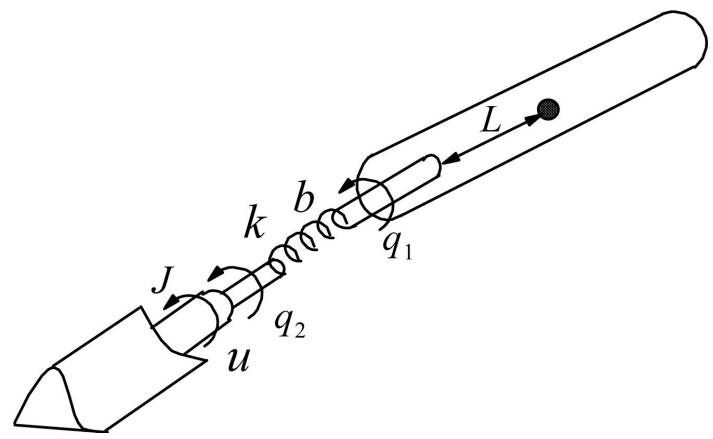

Figure 3. A flexible one link robot. 


$$
\begin{aligned}
w= & \left(1 / L_{g} T_{4}\right)\left(r-L_{f} T_{4}\right) \\
= & \frac{I J}{k_{e}}\left(r-\frac{M g l}{I} \sin x_{1}\left(x_{2}^{2}+\frac{M g l}{I} \cos x_{1}+\frac{k_{e}}{I}\right)\right. \\
& \left.+\frac{k_{e}}{I}\left(x_{1}-x_{3}\right)\left(\frac{k_{e}}{I}+\frac{k_{e}}{J}+\frac{M g l}{I} \cos x_{1}\right)\right)
\end{aligned}
$$

For the system (46) with joint flexibility and damping, i.e., $k_{e}>0, b>0$, it can be verified that it has a relative degree of three and can only be partially feedback linearized. With

$$
\begin{aligned}
& z_{1}=T_{1}(x)=x_{1} \\
& z_{2}=T_{2}(x)=x_{2} \\
& z_{3}=T_{3}(x)=-\frac{M g l}{I} \sin x_{1}-\frac{k_{e}}{I}\left(x_{1}-x_{3}\right)-\frac{b}{I}\left(x_{2}-x_{4}\right)
\end{aligned}
$$

the partial feedback linearization control input is

$$
\begin{aligned}
w= & \frac{I J}{b}\left(r+\frac{M g l}{I} x_{2} \cos x_{1}-\frac{b}{I}\left(\frac{k_{e}}{I}+\frac{k_{e}}{J}\right)\left(x_{1}-x_{3}\right)\right. \\
& \left.+\left(\frac{k_{e}}{I}-\frac{b}{I}\left(\frac{b}{I}+\frac{b}{J}\right)\right)\left(x_{2}-x_{4}\right)\right)
\end{aligned}
$$

which results in the partial linear system $\dot{z}_{1}=x_{2}, \dot{z}_{2}=z_{3}, \dot{z}_{3}=r$. With $z_{4}=T_{4}(x)=x_{3}$ and $T(x)=\left[z_{1}, z_{2}, z_{3}, z_{4}\right]^{\mathrm{T}}, \partial T(x) / \partial x$ is nonsingular, i.e., $T(x)$ is diffeomorphism. It then follows that

$$
\dot{z}_{4}=-\frac{k_{e}}{b} z_{4}+\frac{I}{b} z_{3}+\frac{M g l}{b} \sin z_{1}+\frac{k_{e}}{b} z_{1}+z_{2}
$$

which is a bounded input, bounded state stable zero dynamic system.

In both cases, the control signal $w$ is applied to a fuzzy logic deadzone compensator for the deadzone nonlinearity $u=N(v)$. Some simulation results for fuzzy logic deadzone compensation of the system (46) with deadzone widths $d_{+}=0.2, d_{-}=-0.2$ are given in Figure 1 for the joint flexibility case. The system parameters are as follows: $M=1[\mathrm{~kg}], g=9.8[\mathrm{rad} / \mathrm{sec}], L=1[\mathrm{~m}]$, $I=M L^{2}=1.5\left[\mathrm{~kg} \cdot \mathrm{m}^{2}\right], \quad J=0.2\left[\mathrm{~kg} \cdot \mathrm{m}^{2}\right], \quad k_{e}=10[\mathrm{~N} \cdot \mathrm{m} / \mathrm{rad}]$, $b=0.07\left[\mathrm{~kg} \cdot \mathrm{m}^{2} / \mathrm{sec}\right]$. The tracking response without and with deadzone nonlinearity is shown in Figure 4(a) and Figure 4(b) for $r_{d}=\sin (2 \pi t / 1800)$. The response with a fuzzy deadzone compensator is shown in Figure 4(c), showing significiant performance improvement. Figure 4(d) shows estimates of deadzone widths. Simulation results for the joint flexibility plus damping case are shown in Figure 5 for $r_{d}=\sin (2 \pi t / 1800)$. These results indicates that the fuzzy deadzone compensation significantly improves system tracking performance.

\section{Conclusion}

A fuzzy logic deadzone compensator has been proposed for feedback linearizable nonlinear systems. The classification property of fuzzy logic systems makes them a natural candidate for offsetting this sort of actuator nonlinearity having a 


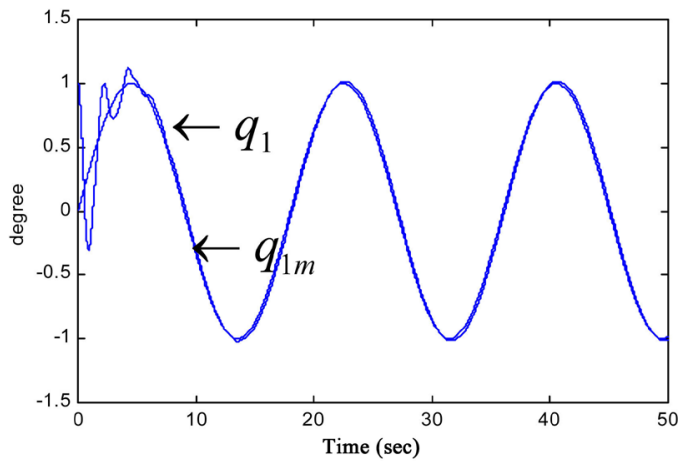

(a)

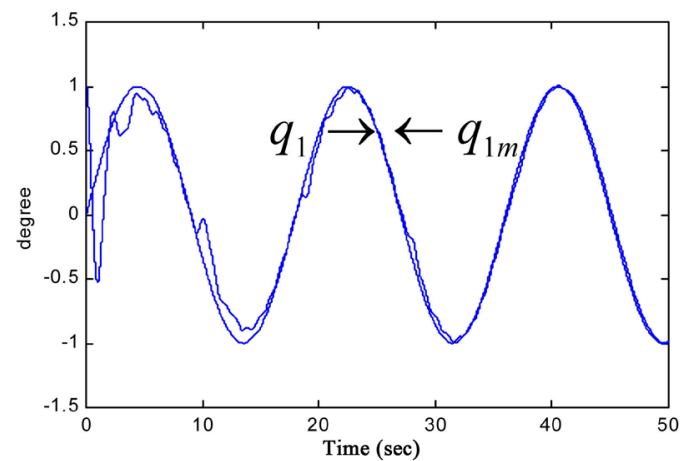

(c)

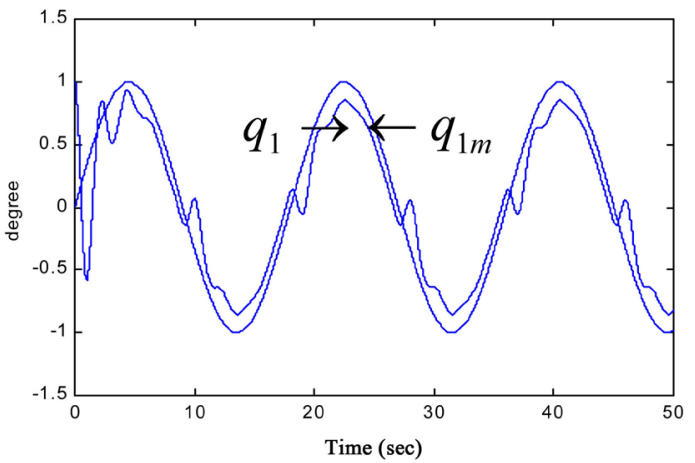

(b)

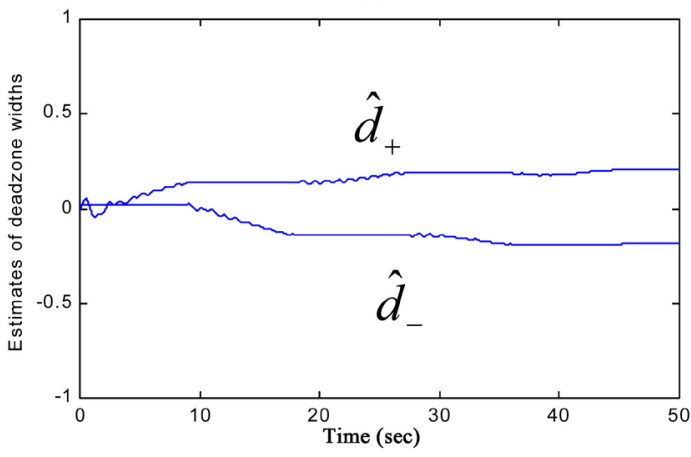

(d)

Figure 4. System response with flexibility (a) with deadzone nonlinearity, (b) without deadzone nonlinearity, and (c) with fuzy logic deadzone compensation. (d) Estimates of deadzone widths.

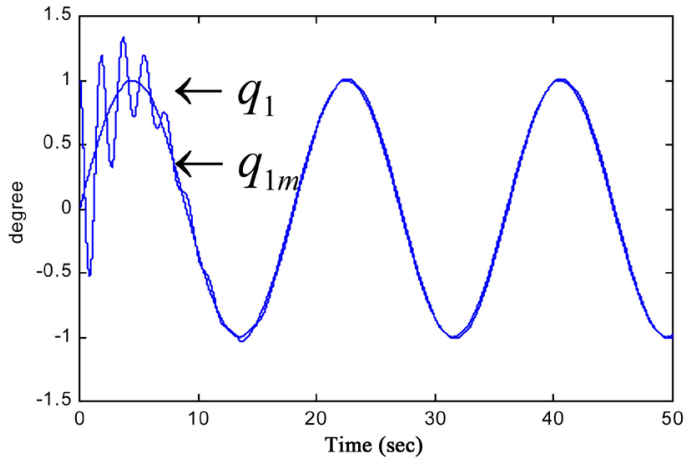

(a)

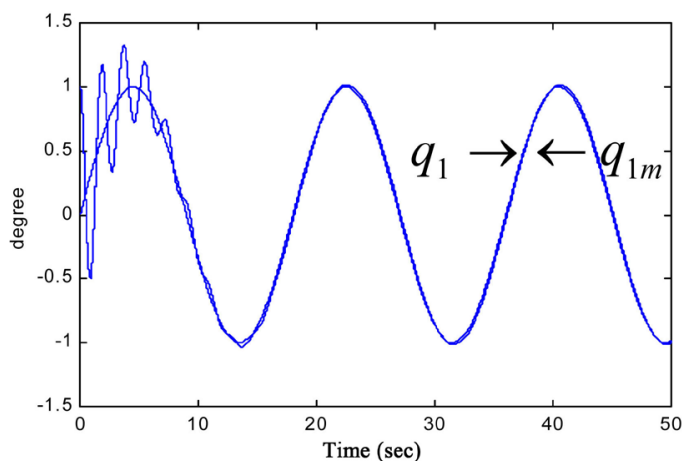

(c)

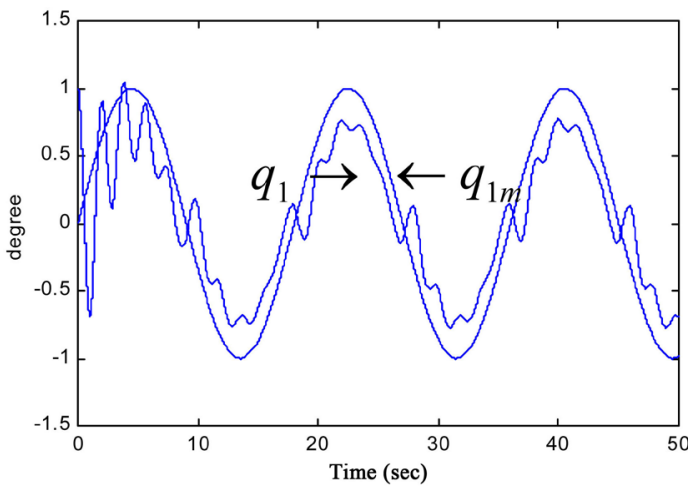

(b)

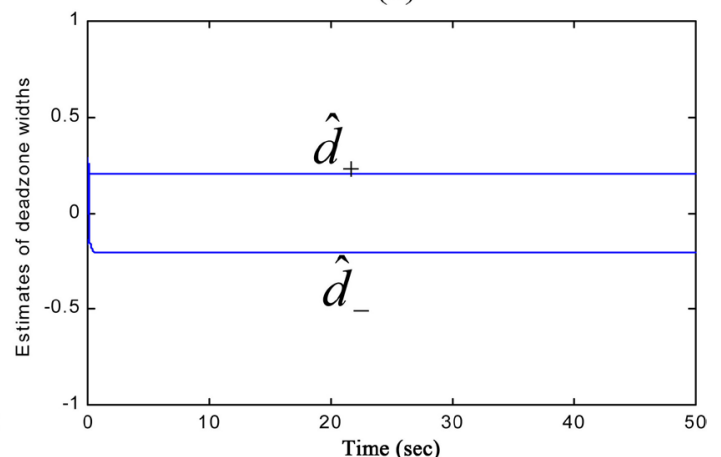

(d)

Figure 5. System response with flexibility and damping (a) with deadzone nonlinearity, (b) without deadzone nonlinearity, and (c) with fuzy logic deadzone compensation, (d) estimates of deadzone widths. 
strong dependence on the region in which the arguments occur. It was shown how to tune the fuzzy logic parameters so that the unknown deadzone parameters are learned on line, resulting an adaptive deadzone compensator. Using nonlinear stability techniques, the bound on tracking error is derived from the tracking error dynamics. Simulation results show that significantly improved system performance can be achieved by the proposed adaptive fuzzy logic compensation. The future research is to get the results of experiment on robot manipulators.

\section{Conflicts of Interest}

The author declares that there is no conflict of interest regarding the publication of this paper.

\section{References}

[1] Xu, Q. (2014) Design and Development of a Compact Flexure-Based XY Precision Positioning System with Centimeter Range. IEEE Transactions on Industrial Electronics, 61, 893-903. https://doi.org/10.1109/TIE.2013.2257139

[2] Chang, C.Y. (2007) Adaptive Fuzzy Controller of the Overhead Cranes with Nonlinear Disturbance. IEEE Transactions on Industrial Informatics, 3, 164-172. https://doi.org/10.1109/TII.2007.898433

[3] Kormushev, P., Deniris, Y. and Caldwell, D.F. (2015) Encoderless Positional Control of a Two Link Robot Manipulator. Robotics and Automation, Seattle, 943-949.

[4] He, W., David, A.S., Yin, Z. and Sun, C. (2016) Neural Network Control of a Robotic Manipulator with Input Dadzone and Output Constraint. IEEE Transactions on Systems, Man, and Cybernetics: Systems, 46, 759-770. https://doi.org/10.1109/TSMC.2015.2466194

[5] Utkin, V.I. (1993) Sliding Mode Control Design Principles and Applications to Electric Drives. IEEE Transactions on Industrial Electronics, 40, 23-36. https://doi.org/10.1109/41.184818

[6] Yu, Z., Ju X. and Ding, Z. (2016) Control of Gear Transmission Servo Systems with Asymmetric Deadzone Nonlinearity. IEEE Transactions on Control Systems Technology, 24, 1472-1479. https://doi.org/10.1109/TCST.2015.2493119

[7] He, W., He, X. and Sun, C. (2017) Vibration Control of an Industrial Moving Strip in the Presence of Input Deadzone. IEEE Transactions on Industrial Electronics, 64, 4680-4689.

[8] Recker, D.A., Kokotovic, P.V., Rhode, D. and Winkelman, J. (1991) Adaptive Nonlinear Control of Systems Containing a Deadzone. IEEE Conference on Decision and Control, Brighton, 11-13 December 1991, 2111-2115. https://doi.org/10.1109/CDC.1991.261510

[9] Tian, M. and Tao, G. (1996) Adaptive Control of a Class of Nonlinear Systems with Unknown Deadzones. IFAC World Congress, San Francisco, 209-214.

[10] Kumar, R. and Kumar, M. (2015) Improvement Power System Stability Using Unified Power Flow Controller Based on Hybrid Fuzzy Logic PID Tuning in SMIB System. Green Computing and Internet of Things, Nodia, October 2015, 815-819. https://doi.org/10.1109/ICGCIoT.2015.7380575

[11] Jang, J.O. (2005) Deadzone Compensation of an XY Positioning Table Using Fuzzy Logic. IEEE Transactions on Industrial Electronics, 52, 1696-1701. 
https://doi.org/10.1109/TIE.2005.858702

[12] Lewis, F.L., Tim, W.K., Wang, L.Z. and Li, Z.X. (1999) Deadzone Compensation in Motion Control Systems Using Adaptive Fuzzy Logic Control. IEEE Transactions on Control Systems Technology, 7, 731-742. https://doi.org/10.1109/87.799674

[13] Oh, S.Y. and Park, D.J. (1998) Design of New Adaptive Fuzzy Logic Controller for Nonlinear Plants with Unknown or Time-Varing Dead Zones. IEEE Transactions on Fuzzy Systems, 6, 481-492.

[14] Lv, M., Baldi, S. and Liu, Z. (2019) The Non-Smoothness Problem in Disturbance Observer Design: A Set Invariance-Based Adaptive Fuzzy Control Method. IEEE Transactions on Fuzzy Systems, 27, 598-604. https://doi.org/10.1109/TFUZZ.2019.2892353

[15] Yang, C., Jiang, Y., Na, J., Li, Z., Cheng, L. and Su, C.Y. (2019) Finite Time Convergence Adaptive Fuzzy Control for Dual Arm Robot with Unknown Kinematic and Dynamics. IEEE Transactions on Fuzzy Systems, 21, 759-770. https://doi.org/10.1109/TFUZZ.2018.2864940

[16] Arif, J., Ray, S. and Chaudhuri, B. (2014) Multivariable Self Tuning Feedback Linearization Controller for Power Oscillation Damping. IEEE Transactions on Control System Technology, 22, 1519-1526. https://doi.org/10.1109/TCST.2013.2279939

[17] Park, H., Chwa, D. and Hong, K.S. (2007) A Feedback Linearization Control of Container Crane: Varying Rope Length. International Journal of Control, Automation and Systems, 5, 379-387.

[18] Isidori, A. (1995) Nonlinear Control Systems. 3rd Edition, Springer-Verlag, Berlin. https://doi.org/10.1007/978-1-84628-615-5

[19] Krstic, M., Kanellakopoulos, I. and Kokotovic, P.V. (1995) Nonlinear and Adaptive Control Design. Wiley, New York.

[20] Slotine, J.J.E. and Li, W. (1991) Applied Nonlinear Control. Englewood Cliffs, Prentice Hall.

[21] Spong, M.W. and Vidyasagar, M. (1989) Robot Dynamic and Control. Wiley, New York. 